\title{
Reflets
}

Revue ontaroise d'intervention sociale et communautaire

\section{D’une loi à l'autre : le système de justice pénale pour les jeunes en Ontario}

\section{Alain Charpentier et Marie Nathalie Moreau}

Volume 6, numéro 2, automne 2000

Problèmes sociaux en Ontario français

URI : https://id.erudit.org/iderudit/026320ar

DOI : https://doi.org/10.7202/026320ar

Aller au sommaire du numéro

Éditeur(s)

Reflets : Revue ontaroise d'intervention sociale et communautaire

ISSN

1203-4576 (imprimé)

1712-8498 (numérique)

Découvrir la revue

Citer cet article

Charpentier, A. \& Moreau, M. N. (2000). D'une loi à l'autre : le système de justice pénale pour les jeunes en Ontario. Reflets, 6(2), 168-181.

https://doi.org/10.7202/026320ar

Tous droits réservés (C) Reflets : Revue ontaroise d'intervention sociale et communautaire, 2000
Ce document est protégé par la loi sur le droit d'auteur. L'utilisation des services d'Érudit (y compris la reproduction) est assujettie à sa politique d'utilisation que vous pouvez consulter en ligne.

https://apropos.erudit.org/fr/usagers/politique-dutilisation/ 


\section{$\mathrm{D}^{\prime}$ une loi à l'autre $\square$ : le système de justice pénale pour les jeunes en Ontario}

Alain Charpentier, M.S.S. et Marie Nathalie Moreau M.C.A. Techniques des services correctionnels, La Cité collégiale, Ottawa

Depuis quelques années, le système canadien de justice pour les jeunes fait l'objet de nombreuses critiques. En mai 1998, le ministère fédéral de la Justice a rendu publique sa stratégie de renouvellement du système de justice pour les jeunes qui comprend trois principaux objectifs : la promotion de la prévention du crime et des solutions de rechange au système officiel de justice, la mise en place de conséquences significatives pour les jeunes contrevenantes et contrevenants et finalement, l'amélioration des efforts déployés dans la réadaptation et la réinsertion sociale des jeunes (ministère de la Justice 1999).

Le remplacement de la Loi sur les jeunes contrevenants (LJC), présentement en vigueur, par la Loi sur le système de justice pénale pour les adolescents (projet de loi C-3), représente une étape importante dans la mise en oeuvre de la stratégie fédérale. Le projet de Loi sur le système de justice pénale pour les adolescents a été réintroduit à la session parlementaire le 14 octobre 1999 et suit depuis lors les étapes laborieuses du processus législatif.

De juridiction fédérale, la Loi sur le système de justice pénale pour adolescents sera cependant administrée par les différentes provinces. L'Ontario mène présentement une campagne de lobbying intensive pour obtenir des changements efficaces dans cette loi fédérale. $M$. Harris a présenté récemment la position de son 
gouvernement sur ce sujet, dans le document intitulé Brueprint for a Safer Ontario : (Canada News Wire 1999 : 1).

Dans ce texte, nous examinerons d'abord les modèles idéologiques en présence dans le débat sur les jeunes contrevenants pour, ensuite, poser un regard sur le système de justice pénal ontarien pour les jeunes en tenant compte de la problématique franco-ontarienne. Finalement, nous mettrons en relief certains changements proposés par la nouvelle loi et nous offrirons des pistes de réflexion quant à la façon d'aborder la problématique de la délinquance juvénile en Ontario.

\section{Les modèles idéologiques en présence}

Reid et Reitsma-Street (1984) distinguent quatre modèles idéologiques pour comprendre les attitudes et les croyances des Canadiennes et des Canadiens face aux solutions à apporter à la problématique de la délinquance juvénile : les modèles de bienêtre, de contrôle du crime, de justice et celui du changement communautaire.

Le modèle de bien-être, qui date de la fin du $19^{\text {ème }}$ siècle, provient de l'école positiviste de criminologie, inspirée par les mouvements sociaux de l'époque. Les adeptes de ce modèle estiment que les jeunes sont les produits de leur environnement et, par conséquent, ne sont pas responsables de leurs actes illégaux :

Les positivistes soutenaient que le comportement antisocial était la manifestation de la réponse de l'individu à un stimulus externe sur lequel celui-ci n'avait aucun contrôle [...][et que] s'il s'agit d'un enfant, [il ou elle] ne peut être tenu responsable de ses actes illégaux et toute peine est donc inefficace, voire injuste (Archambault 1983 : 9).

Si l'imposition d'une peine est jugée inefficace, il faut alors axer les interventions sur la réhabilitation ou le traitement du jeune, 
avec le soutien d'intervenantes et d'intervenants sociaux ayant l'expertise dans ce domaine. Pour prévenir le crime, ce modèle préconise notamment la modification de l'environnement, particulièrement du milieu familial, perçu comme étant une des causes premières de la délinquance. La philosophie de la Loi sur les jeunes délinquants qui a précédé l'actuelle Loi sur les jeunes contrevenants était basée sur ce modèle qui se fondait d'abord et avant tout sur le bien-être de l'enfant.

À l'autre extrême, les adhérents au modèle de contrôle du crime imputent à l'État et aux tribunaux la responsabilité du contrôle de la criminalité, du maintien de l'ordre et de la liberté morale. Selon eux, le système judiciaire doit punir les comportements criminels afin d'assurer la protection de la société. Puisque les comportements délinquants des jeunes sont perçus comme étant menaçants pour la société, ceux-ci doivent être tenus responsables de leurs actes et leurs parents peuvent partager cette responsabilité si l'État juge que cette mesure contribuera à diminuer la criminalité.

Situé à mi-chemin entre les modèles de bien-être et du contrôle du crime, le modèle de justice s'inspire de l'école classique de criminologie. Le juge Archambault (1983), parrain de la LJC, souligne que cette école a inspiré la réforme du système de justice pour les jeunes en mettant l'accent sur leurs droits et leurs responsabilités. Les jeunes sont responsables de leurs actes délinquants, mais à un niveau moindre que les adultes, en raison de leur âge et de leur degré de maturité. Les sanctions doivent donc être moins sévères que celles infligées aux adultes. Ce modèle préconise à la fois la protection de la société et la réhabilitation du jeune. Par ailleurs, on attribue la responsabilité première au jeune et par conséquent, ses parents ne sont pas jugés responsables de ses actes.

D'autre part, le modèle du changement communautaire est basé sur le marxisme. Les politiques issues de ce modèle viseront, notamment, à changer les facteurs sociaux menant à l'inégalité, à la pauvreté et à la délinquance. Puisqu'une restructuration de la société serait nécessaire pour atteindre cet objectif ultime, les adhérents de ce modèle proposent un certain nombre d'étapes intermédiaires telles qu'un contrôle communautaire accru grâce 
à un processus de décentralisation des décisions et une plus grande implication des citoyennes et des citoyens dans la prévention et le contrôle de la criminalité. Caputo (1987 :140) explique les façons dont les communautés peuvent se prendre en main :

Lorsque les communautés développent une identité collective et un but commun, elles ont la capacité de définir et de résoudre leurs problèmes. Elles développent davantage leur réseau organisationnel et acquièrent plus de contrôle sur les ressources institutionnelles et communautaires (traduction libre).

Pour les adhérents au modèle de changement communautaire, l'environnement familial est façonné par les politiques gouvernementales et par les structures sociales inéquitables. Par conséquent, la famille n'est pas perçue comme étant responsable de la délinquance du jeune, mais plutôt comme victime d'une société qui perpétue les problèmes sociaux tels que la pauvreté, le chômage, et les institutions abusives et autoritaires.

La juxtaposition des quatre modèles idéologiques dans la déclaration de principes de la LJC a permis de faire les compromis nécessaires pour que celle-ci soit adoptée en 1982 et mise en vigueur en 1984 (Reid et Reitsma-Street 1984). Par contre, ce n'est que suite à de nombreux débats que l'Ontario l'a mise en application en 1985 (Moreau 1991).

\section{Le système de justice pour jeunes contrevenantes et contrevenants en Ontario}

Les provinces ont beaucoup de latitude pour administrer la LJC, qui s'applique aux jeunes de 12 à 18 ans moins un jour.

L'Ontario a adapté le système de justice pénal déjà existant pour se conformer aux nouvelles exigences de la LJC. Le ministère des Services sociaux et communautaires, responsable déjà des jeunes contrevenantes et contrevenants de 12 à 15 ans, a continué 
à desservir cette clientèle en consolidant les services déjà offerts. Par ailleurs, les jeunes de 16 à 18 ans, considérés jusque là comme étant adultes en Ontario, sont maintenant de jeunes contrevenantes et contrevenants sous la responsabilité du ministère des Services correctionnels. Ce ministère a dû développer des programmes et des services pour cette clientèle, donnant ainsi naissance à un système comportant deux phases : la première regroupe les jeunes contrevenantes et contrevenants de 12 à 15 ans et la deuxième regroupe ceux et celles de16 à 18 ans moins un jour.

Le ministère des Services sociaux et communautaires a traditionnellement adopté le modèle idéologique du bien-être tel que décrit dans la Loi sur les services à l'enfance et à la famille: "cette loi a][...] comme objectif principal, [de] promouvoir l'intérêt véritable de l'enfant, sa protection et son bien-être " (Loi sur les services à l'enfance et à la famille 1999 :art. 1). Il est à noter que cette dernière loi s'applique aussi aux jeunes contrevenantes et contrevenants de phase I. Récemment, ce ministère a adopté un discours en faveur du modèle idéologique du changement communautaire: « favoriser l'autonomie et compléter, plutôt que remplacer, les formes de soutien traditionnelles (famille, amis et collectivité). " (ministère des Services sociaux et communautaires $1997: 3$ ).

De son côté, le ministère des Services correctionnels adopte une position axée davantage sur le modèle du contrôle du crime. L'objectif premier est la protection de la société. La mission de ce ministère indique clairement l'importance accordée à l'incarcération : "The primary mission of our Ministry is to provide custody, community supervision, and information/reports as directed by the courts, and as provided for in federal and provincial legislation governing correctional services in Ontario. " (Ministry of Correctional Services 1993 : 0101 02). D'ailleurs, l'Ontario est l'une des provinces canadiennes où l'on incarcère le plus de jeunes pour des délits violents et non violents. (Centre canadien de la statistique juridique 1997).

Les pratiques ontariennes se traduisent, entre autres, par deux systèmes indépendants de tribunaux, de probation et de milieux résidentiels, comme l'illustrent les exemples suivants. Les dossiers des jeunes de phase I sont confiés à la division familiale de la cour 
provinciale, tandis que la division criminelle s'occupe des dossiers des jeunes de phase II. Les agentes et agents de probation supervisent donc à la fois des jeunes de phase II et des adultes. La dualité ministérielle est d'autant plus marquée par l'existence d'une catégorie de jeunes contrevenantes et contrevenants de niveau 1 créée par le ministère des Services correctionnels de l'Ontario. Les jeunes de ce groupe ont commis des délits avec violence et sont considérés comme étant à risque de récidive. Par conséquent, pour ceux qui sont placés sous garde en milieu résidentiel, les privilèges de sortie en communauté sont très limités. Ce système d'étiquetage n'est pas utilisé pour les jeunes de phase I.

Les problèmes concrets occasionnés par l'existence de deux juridictions ministérielles indépendantes l'une de l'autre sont nombreux, notamment : un traitement différentiel accordé aux jeunes, un manque de communication entre les deux ministères et un manque de continuité dans la prestation de services.

\section{La situation des jeunes Franco-Ontariennes et Franco-Ontariens dans le système de justice pénale}

Bien que la Loi sur les services en français permette de recevoir des services en français dans les régions désignées, quelles en sont la qualité et la disponibilité dans le système de justice pénale s'adressant aux jeunes?

Dans la région d'Ottawa-Carleton, Martin et Gaulin (1995) soulignent que tous les rapports de police sont rédigés seulement en anglais. Selon un policier des services jeunesse, cette pratique s'explique par le faible nombre de jeunes francophones unilingues : " il y a eu seulement 2 ou 3 jeunes qui parlaient uniquement le français au cours des 10 dernières années " (traduction libre) (Martin et Gaulin 1995 : 17). Ainsi, on prend pour acquis que les jeunes qui sont bilingues n'ont pas besoin de recevoir des services en français. 
Les Franco-Ontariennes et les Franco-Ontariens ont aussi le droit de recevoir des services en français dans les tribunaux. Par contre, seulement $20 \%$ des jeunes en font la demande et, de ce groupe, les deux tiers reçoivent, malgré tout, des services en anglais (Cousineau 1994 dans Martin et Gaulin 1995). Les principales raisons évoquées pour expliquer cette situation sont l'absence de juges francophones et l'aisance des avocates et des avocats francophones qui préferent travailler en anglais.

En milieu résidentiel, les intervenants doivent demander aux jeunes si elles ou ils désirent recevoir des services en français seulement lorsque l'organisme se situe dans une région désignée (Ministry of Correctional Services 1993 : 0103 07). Les services en français sont alors offerts en fonction de la capacité linguistique des intervenants sur place au moment de la demande. Dans les régions non désignées, deux possibilités s'offrent aux jeunes : ils peuvent soit être transférés dans une ville qui offre des services en français ou recevoir des services uniquement en anglais.

Par ailleurs, les mécanismes de surveillance de l'application de la Loi sur les services en français dans les milieux pour jeunes contrevenantes et contrevenants sont quasi absents. Il faut alors se demander si les jeunes Franco-Ontariennes et Franco-Ontariens reçoivent vraiment les services auxquels ils ont droit dans leur langue maternelle.

\section{L'impact de l'implantation de la Loi sur le système de justice pénale pour adolescents}

La présence de modèles idéologiques divergents dans l'application de la LJC ne peut que provoquer la critique. Le projet de Loi sur le système de justice pénale pour adolescents propose des changements ayant pour but de répondre aux attentes des adhérents de trois des quatre différents camps.

Le modèle de justice se dégage clairement dans la déclaration de principes du projet de loi C-3 : «le système de justice pénale 
pour les adolescents doit être distinct de celui pour les adultes et mettre l'accent sur : une responsabilité juste et proportionnelle, compatible avec leur état de dépendance et leur degré de maturité [...]" ministère de la Justice 2000b : art. 3).

Le modèle de bien-être semble cependant absent dans ce projet de loi. Il est impossible, soit dans le préambule ou dans la déclaration de principes, d'identifier une position en faveur du bien-être des jeunes, contrairement à la LJC qui précise notamment que : «[...] leur degré de développement et de maturité crée des besoins spéciaux qui exigent conseils et assistance" (Loi sur les jeunes contrevenants 1995 : art.3). Alors comment le ministère des Services sociaux et communautaires continuera-t-il à " promouvoir l'intérêt véritable ", la "protection" et le "bienêtre » des jeunes contrevenantes et contrevenants de 12 à 15 ans, tel que stipulé dans la déclaration de principes de la Loi sur les services à l'enfance et à la famille de l'Ontario?

Par contre, les modèles du contrôle du crime et de changement communautaire prennent beaucoup d'ampleur dans le projet de loi C-3.

D'abord, le modèle du contrôle du crime influence plusieurs aspects du projet de loi qui favorise « l'imputabilité, la responsabilité et les conséquences significatives " (ministère de la Justice 2000a : 3). La déclaration de principes stipule clairement que l'objectif premier du projet de loi est la protection de la société : « le système de justice pénale pour adolescents a pour but premier de protéger le public [...]» (ministère de la Justice 2000b : article 3). Voici quelques exemples d'applications pratiques de cette loi :

- un jeune de 14 ans et plus reconnu coupable d'un délit punissable chez l'adulte d'emprisonnement de plus de deux ans serait passible d'une peine d'adulte;

- un jeune de 14 ans et plus reconnu coupable de meurtre, de tentative de meurtre, d'homicide involontaire ou d'agression sexuelle grave, serait condamné automatiquement à une peine d'adulte;

- un jeune qui atteint l'âge de 20 ans alors qu'il se trouve en mise sous garde dans un milieu pour adolescents, devrait finir de purger sa peine dans un milieu carcéral pour adultes (ministère de la Justice 2000a : 5-6). 
Le projet de loi crée une catégorie réservée aux jeunes qui commettent des délits violents. Selon les adhérents à la position du contrôle du crime, la protection de la société est mieux assurée lorsque ces jeunes sont incarcérés afin de contrôler leur criminalité. Cela renforce d'ailleurs la direction adoptée par le ministère ontarien des Services correctionnels pour les jeunes de phase II.

Bien que l'idéologie du contrôle du crime soit évidente dans l'esprit du projet de loi, certains groupes militent pour un contrôle accru. C'est le cas notamment de l'Honorable Frank Kleek, ministre sans porte-feuille, qui présente la résolution suivante à l'Assemblée législative de l'Ontario le 15 mai 2000 :

"Que L'assemblée législative de l'Ontario :

(a) condamne la faiblesse de la Loi sur les jeunes contrevenants et demande de la remplacer par une loi plus sévère qui rend les jeunes responsables de leurs actions;

(b) rejette le projet de loi C-3 qui ne tient pas suffisamment compte des préoccupations des membres de la communauté;

(c) rejette tout changement qui rendrait la loi encore plus faible;

(d) rejette les tentatives du gouvernement fédéral de raccourcir les peines d'incarcération des jeunes ayant commis un délit criminel;

(e) croit qu'un jeune de 16 ou 17 ans ayant commis un délit sérieux devrait automatiquement être jugé devant un tribunal pour adultes;

(f) croit qu'un jeune qui commet des délits violents devrait recevoir une peine d'adulte" (Hansard, mai 2000).

Ce qui est inquiétant dans cette résolution, c'est qu'on n'y mentionne aucunement le meilleur intérêt des jeunes. Pourtant, le docteur Parker Loewen, défenseur des droits des enfants pour la province du Saskatchewan, nous rappelle qu'un pourcentage élevé de jeunes qui commettent des délits sont victimes de pauvreté, de négligence, de violence physique et sexuelle (Chapman 1998). Certes, la protection des gens de la communauté est essentielle, mais non pas au détriment de la réadaptation des jeunes. Le 
préambule du projet de loi reconnait la Convention des Nations Unies relative aux droits de l'enfant (ministère de la Justice 2000b). Par contre, la loi n'indique pas de quelle façon les provinces doivent la prendre en considération. Le gouvernement ontarien pourra donc ignorer les besoins des jeunes sur les plans de la prévention et de la réadaptation.

D'autre part, ce qui est encourageant dans le projet de loi C-3, c'est que la communauté prend une part plus active dans la mise en place de mécanismes pour réduire la délinquance chez les jeunes. Le modèle de changement communautaire prend ainsi de l'importance et se reflète dans la déclaration de principes : «[...] la prévention du crime par la suppression des causes sous-jacentes à la criminalité chez les adolescents [...] [et] faire participer leur père et mère, leur famille étendue, les membres de leur collectivité et certains organismes sociaux ou autres à leur réadaptation et leur réinsertion sociale [...]" (ministère de la Justice 2000b : article 3).

Présentement, le gouvernement ontarien injecte 3 millions de dollars dans la création de comités de justice (Hansard, mai 2000). Ceux-ci sont composés de membres de la communauté et sont en quelque sorte des tribunaux qui doivent entendre la cause du jeune et imposer une sanction. Les causes entendues impliquent des jeunes qui ont commis des délits de nature non violente. Le processus vise à impliquer la communauté et notamment les parents de jeunes contrevenantes et contrevenants, qui sont souvent blâmés ou ignorés par le système judiciaire (Charpentier 1997). À la lumière des résultats de recherches contemporaines portant sur la délinquance juvénile, la pertinence de l'implication des parents de jeunes contrevenantes et contrevenants ne fait plus de doute : "Les causes de la délinquance—et ses solutions- - sont bien plutôt le résultat d'une combinaison de facteurs individuels, familiaux et sociaux. Les stratégies d'intervention doivent être axées non seulement sur l'individu, mais aussi sur son environnement et notamment sa famille " (Groupe de travail fédéral-provincial-territorial $1996: 475)$.

Une approche similaire, soit les conférences familiales, a fait ses preuves en Nouvelle-Zélande : « Le nombre des placements sous garde visant les jeunes contrevenants a diminué de moitié 
l'année qui a suivi l'adoption des modifications législatives et ces chiffres sont demeurés depuis à ce nouveau niveau " (Groupe de travail fédéral-provincial-territorial sur la justice applicable aux jeunes 1996 :209). Cette approche liée au modèle de changement communautaire permet à la communauté de participer à l'élaboration de solutions créatives et ce, dans le meilleur intérêt des jeunes. Lorsque cette finalité est atteinte, les jeunes et la communauté en ressortent gagnants.

En somme, les comités de justice présentent une avenue viable. Il serait cependant souhaitable qu'ils puissent transiger non seulement avec les jeunes qui ont commis des délits non violents, mais aussi avec celles et ceux qui s'engagent dans des activités criminelles violentes, celles-ci étant souvent dirigées envers des membres de la communauté.

\section{L'absence de balises claires pour la promotion des services en français}

Le projet de loi C-3 ne propose pas de balises claires pour la prestation de services en français destinés aux jeunes contrevenantes et contrevenants.

La déclaration de principes stipule que "les mesures visant à régler le problème de la criminalité chez les jeunes doivent [...] prendre en compte $[\ldots]$ les différences ethniques, culturelles et linguistiques [...] " (ministère de la Justice 2000b : article 3). Le projet de loi fait référence à des différences linguistiques et culturelles mais il n'indique pas de quelle façon la province doit prendre en compte ces différences. D'ailleurs, on évite de nommer spécifiquement le français qui pourtant est une langue officielle au Canada. Cette clause ressemble étrangement à ce que nous propose la Loi sur les services en français ontarienne qui est une loi dépourvue de poids.

Il faut se demander quelle portion des $\$ 206$ millions injectés par le gouvernement fédéral (ministère de la Justice 2000b) sera 
donnée au développement de programmes pouvant répondre aux besoins des jeunes Franco-Ontariennes et Franco-Ontariens en conflit avec la loi.

\section{Conclusion}

Le projet de Loi sur le système de justice pénale pour adolescents (projet de loi C-3), comme la LJC, est issu de compromis idéologiques divergents. Malgré la présence du modèle de justice dans ce projet de loi, deux pôles idéologiques opposés prennent de l'ampleur, soit les modèles de contrôle du crime et du changement communautaire. Par conséquent, le projet de loi C-3 laisse beaucoup de latitude aux provinces quant à son application.

En Ontario, l'idéologie du contrôle du crime est bien ancrée dans l'opinion publique et cela se traduit dans les prises de position des politiciennes et des politiciens. Les approches issues de cette idéologie sont axées sur la punition des jeunes contrevenants et non sur leur réadaptation. Par ailleurs, le modèle du changement communautaire mise sur la participation des membres de la communauté afin de trouver des solutions au problème de la délinquance chez les jeunes. Ce modèle offre ainsi le meilleur cadre pour favoriser la réadaptation et la réinsertion sociale des jeunes contrevenantes et contrevenants.

Il est difficile d'établir avec exactitude l'impact de la Loi sur le système de justice pénale pour adolescents sur la délinquance juvénile en Ontario, sans connaitre son application pratique. Par contre, si le présent laisse présager l'avenir, le traitement différentiel des jeunes ainsi que le manque de communication et de continuité dans la prestation de services au sein de deux ministères risquent de se perpétuer.

En ce qui concerne les Franco-Ontariennes et FrancoOntariens, la situation n'ira guère en s'améliorant, compte tenu de la faiblesse de la déclaration de principes sur la langue et du traitement actuel des jeunes contrevenantes et contrevenants 
francophones en Ontario. Pour que l'on puisse répondre adéquatement aux besoins de ce groupe, il faudra d'abord qu'il y ait une volonté politique ferme de la part du gouvernement Harris et l'injection de fonds, afin d'améliorer la surveillance de l'application des services en français.

Finalement, chaque citoyenne et chaque citoyen adhère à un ou à plusieurs modèles idéologiques et, à travers ses attitudes et croyances, juge de la pertinence du projet de Loi sur le système de justice pénale pour les adolescents. Certes, il est important que toutes et tous se sentent en sécurité dans leur communauté mais n'estil pas aussi fondamental que cette même communauté investisse dans ses jeunes citoyennes et citoyens?

\section{Bibliographie}

ARCHAMBAULT, Omer (1983). "Philosophie et principes de la loi sur les jeunes contrevenants ", Journal des juges provinciaux, vol. 7, no 1, 8-34.

CANADA, CENTRE CANADIEN DE LA STATISTIQUE JURIDIQUE (1997). Statistiques sur la criminalité 1996-1997, Ottawa, Statistique Canada.

CANADA, MINISTÈRE DE LA JUSTICE (2000a). Initiative de renouvellement du système de justice pour les jeunes. Une nouvelle loi, une nouvelle approche, Ottawa, ministère de la Justice.

CANADA, MINISTÈRE DE LA JUSTICE (2000b). La loi sur le système de justice pénale pour les adolescents, Ottawa, ministère de la Justice, adresse Internet : http://www.canada.justice.gc.ca/ $\mathrm{fr} / \mathrm{min} / \mathrm{pub} / \mathrm{sjpa} /$ youth.html.

CANADA, MINISTÈRE DE LA JUSTICE (1999). L'initiative de renouvellement du système de justice pour les jeunes. Une nouvelle loi, une nouvelle approche, Bulletin no 1, Ottawa, ministère de la Justice.

CANADA NEWS WIRE (mai 1999). Harris tells parolees "If you're doing crack, you're going back ", adresse Internet : http ://www.newswire.ca/releases/May1999/17/c4634.html.

CAPUTO,T.C. (1987). « The Young Offender Act : Children's Rights, Children's Wrongs », Canadian Public Policy, vol. 13, no 2, 125-143.

CHAPMAN, Sharon (oct.1998). Stop Blaming Youth, Say Provincial Children's Advocates, gouvernement de la Saskatchewan, Children's Advocate Office, adresse Internet : http://www.gov.sk.ca/newsrel/ 1998Oct/823.98102904.html.

CHARPENTIER, Alain (1997). L'empowerment : le défi des intervenantes et des intervenants auprès des parents de jeunes contrevenants de 12 à 15 ans dans la région d'Ottawa, mémoire de maîtrise, École de service social, Université d'Ottawa.

GROUPE DE TRAVAIL FÉDÉRAL-PROVINCIAL-TERRITORIAL (1996). Examen de la Loi sur les jeunes contrevenants et du système de justice applicable aux jeunes au Canada, Ottawa, ministère de la Justice.

Loi sur les jeunes contrevenants (1995), L.R.C. 1985, chap.Y-1. 
Loi sur les services à l'enfance et à la famille (1999), L.R.O., 1990, chap. 19.

MARTIN, Carole et Louise GAULIN (1995). Preliminary Study on the Statistical Information Available on Francophone Young Offenders in Eastern Ontario, Ottawa, doc. mineo.

MOREAU, Marie Nathalie (1991). An Analysis of Programming Offered in Secure Custody for Phase II Young Offenders, mémoire de maîtrise, Département de criminologie, Université d'Ottawa.

ONTARIO, HANSARD (mai 2000), 1ère session parlementaire, 37ème législature, le 15 mai, adresse Internet : http ://www.ontla.on.ca/hansard/hansardindex.htm.

ONTARIO, MINISTÈRE DES SERVICES SOCIAUX ET COMMUNAUTAIRES (1997). Pour des services au service des gens. Nouveau cadre de prestation des services à l'enfance et aux personnes ayant un handicap de développement, Toronto, Imprimeur de la reine.

ONTARIO, MINISTRY OF CORRECTIONAL SERVICES (1993). YOA Operational Policy and Procedures Manual, doc. mineo.

REID, Susan A. et Marge REITSMA-STREET (1984). "Assumptions and Implications of New Canadian Legislation for Young Offenders», Canadian Criminology Forum, vol. 7, no 1, 510-532. 